\title{
Gênero e saúde no Brasil: considerações a partir da Pesquisa Nacional por Amostra de Domicílios*
}

\section{Gender and health in Brazil: considerations based on the National Household Sampling Survey}

\author{
Estela M.L. Aquino*, Grejce M.S. Menezes Marúcia B. Amoedo
}

\begin{abstract}
AQUINO, E.M.L et al. Gênero e saúde no Brasil: consideraçōes a partir da Pesquisa Nacional por Amostra de Domicllios. Rev. Saúde públ., S. Paulo, 26: 195-202, 1992. A mulher brasileira tem vivido mais que o homem, como ocorre em países industrializados centrais. Nesses países, paradoxalmente, as mulheres apresentam indicadores de morbidade mais altos que os homens. O conhecimento sobre o padrão nacional pode ajudar a compreender os determinantes de sua própria realidade, permitindo antecipar tendências futuras e adequar os serviços de saúde. Com esta perspectiva foi feito um estudo de morbidade, a partir de dados da Pesquisa Nacional por Amostra de Domicllios (PNAD/IBGE) de 1986, em dez Estados brasileiros, construindo-se coeficientes de prevalência de morbidade, de demanda e de utilização de serviços segundo sexo, e padronizados por idade pelo método direto. Como medida dos diferenciais, usou-se razões entre os sexos. A sobremorbidade feminina foi constante -em todas as regiōes. Os diferenciais de uso de serviços apresentaram variação regional, sugerindo relação com a oferta de serviços de saúde. Os diferenciais foram nulos na infância; assumiram seus mais altos valores na idade reprodutiva das mulheres, diminuindo depois dos 60 anos. $O$ padrão foi quase constante em todo o pás. Parte do fenómeno pode ser explicada por fatores de ordem metodológica. Contudo, os resultados foram semelhantes aos de outros países. As transformações profundas no padrāo reprodutivo e na inserção social da mulher brasileira têm seu impacto sobre a saúde e o consumo de serviços ainda não avaliado. Recomenda-se a realização de estudos mais específicos que contribuam para a reorganização do sistema de saúde de modo equânime e universal.
\end{abstract}

Descritores: Saúde da mulher. Morbidade. Necessidades e demanda de serviços de saúde, tendências.

\section{Introdução}

As mulheres brasileiras têm progressivamente vivido mais do que os homens. Em 1980, a esperança de vida ao nascer de crianças do sexo feminino já se situava em torno de sete anos mais do que aquela estimada para as do sexo masculino ${ }^{13}$.

O fenômeno é semelhante ao que vem ocorrendo em países industrializados centrais $^{18,19,21}$, onde o principal responsável pela sobremortalidade masculina é o grupo de doenças do aparelho circulatório 15,20 . Na realidade

* Apresentado no I Congresso Brasileiro de Epidemiologia (ABRASCO/UNICAMPO), Campinas (SP) setembro de 1990.

** Instituto de Medicina Social da Universidade do Estado do Rio de Janeiro - Rio de Janeiro, RJ - Brasil; Núcleo de Estudos Mulher e Saúde do Departamento de Medicina Preventiva da Universidade Federal da Bahia (DMP/UFBA) - Salvador, BA - Brasil.

*** Núcleo de Estudos Mulher e Saúde (DMP/UFBA).

**** Graduanda em Medicina, bolsista de iniciaçảo científica do CNPq - Salvador, BA - Brasil.

Separatas/Reprints: E.M.L. Aquino - Rua Padre Feijó, 29 42 andar - Canela - 40110-170 - Salvador, BA - Brasil. brasileira, estas contribuem, de maneira relevante, para a ocorrência do mesmo fenômeno, pela grande magnitude que assumem como causas de morte, ainda que apresentando relativamente pequenas diferenças entre os sexos. De fato, a sobremortalidade masculina tem se dado principalmente às custas das causas violentas de morte, que acometem mais os homens e que têm apresentado niveis muito altos e crescentes ${ }^{2}$.

A literatura internacional, relativa a países capitalistas centrais, tem registrado um aparente paradoxo, que se traduz no fato das mulheres, embora morrendo menos que os homens, em praticamente todas as faixas etárias, apresentarem indicadores de morbidade e de utilização de serviços de saúde mais elevados.

Parece existir uma unanimidade quanto à maior ocorrência, entre as mulheres, de doenças agudas e das incapacidades advindas destas condiçōes, como a restrição de atividades habituais e a necessidade de permanência no leito $14,15,18,20$, mesmo quando excluídas as complicaçōes ligadas à gravidez e ao parto ${ }^{15}$. Entre as doenças que freqüentemente contribuem para 
este excesso feminino de problemas de saúde, estão as doenças infecciosas, as condiçōes respiratórias, as doenças do sistema digestivo e outras condiçōes agudas, como as dores de cabeça e de ouvido, problemas de pele, viroses inespecificas e problemas musculo-esqueléticos ${ }^{17}$. Excetua-se, de modo marcante, o grupo de lesões por causas externas, as quais säo mais freqüentes em indivíduos do sexo maculino ${ }^{17,20}$.

As mulheres também costumam apresentar mais incapacidades advindas de condiçōes agudas como, por exemplo, reduzindo suas atividades usuais de 12 a $35 \%$ mais que os homens, e tendendo a permanecer mais dias ao leito quando adoecem ${ }^{17}$.

Em relaçāo às doenças crônicas, entre crianças e adolescentes, os indivíduos do sexo masculino têm relato mais freqüente de problemas. Em adultos, o padrão assemelha-se ao das doenças agudas, com as mulheres relatando maior ocortência de problemas crônicos do que os homens ${ }^{17}$. Todavia, quanto às incapacidades impostas por essas condições, em todas as idades, especialmente em maiores de 65 anos de idade, os homens têm maior propensão à maior parte das limitações, como a restrição ao exercício de sua atividade principal. Um indicador de severidade, ainda mais expressivo - a limitação da locomoção - costuma ser mais encontrado em homens, entre 15 e 64 anos. Contudo, acima desta idade, a perda de locomoção é mais comum entre as pessoas do sexo feminino.

A sobreutilização feminina de serviços de saúde, sejam estes ambulatoriais ou hospitalares, também é constante na literatura. $E$ enquanto a demanda masculina por serviços ambulatoriais é descrita, em sua maior parte, como gerada pelo trabalho ou pelo seguro social, a demanda feminina apresenta-se como essencialmente voluntária', revelando uma maior propensão das mulheres a buscarem cuidados de saúde de modo espontâneo.

As maiores taxas femininas de hospitalização permanecem mesmo padronizando-se a idade. Todavia, excluindo-se as condiçōes obstétricas, a diferença reduz-se substancialmente ${ }^{12}$. Se além dessas, forem excluídas ainda aquelas condições que ocorrem exclusivamente em cada sexo (doenças da próstata e desordens do aparelho reprodutor feminino, por exemplo), os diferenciais virtualmente desaparecem ${ }^{15,18}$.

Dentre as explicações para esse padrão estariam, de um lado, diferenças biológicas, com as mulheres apresentando maior resistência a doenças, conferida por fatores genéticos e hormonais. Estas explicaçōes seriam razoáveis quanto à mortalidade por algumas doenças, como as cardiovasculares, mas nāo são consistentes com o fato das mulheres relatarem mais problemas de saúde do que os homens.

Os diferenciais encontrados têm sido em parte atribuídos a exposição diferenciada a fatores de tisco, especialmente no trabalho, como decortência do lugar social de homens e mulheres. Além disso, diferenças de gênero na construção da experiência de adoecimento - tanto na percepçāo, quanto no relato diferenciado entre os sexos - também contribuiriam para a existência desses diferenciais de morbidade e de utilização de serviços de saúde.

No Brasil, embora em quase todos os trabalhos da área de saúde os indivíduos estudados sejam categorizados quanto ao sexo, as diferenças encontradas tendem a ser naturalizadas e tratadas tão somente em sua dimensão biológica.

Com o presente estudo, pretende-se conhecer o padrāo brasileiro de morbidade e de utilização de serviços quanto aos diferenciais por sexo nas diversas faixas etárias e, à luz das recentes e profundas mudanças na situação social da mulher, obter indicações sobre tendências futuras.

Admitiu-se como pressuposto teórico o caráter essencialmente social das diferenças baseadas no sexo; na reafirmação do social não excluiu-se a dimensão biológica, mas, para uma análise mais profícua, incorporou-se a noção de gênero, contextualizada historicamente ${ }^{16}$. Implicitamente a essa categoria, também está a compreensão de que ser mulher e ser homem definem-se de modo recíproco, sendo o estudo sobre a situação de saúde de um dos sexos, necessariamente enriquecido pela comparação a do outro.

Mulheres e homens tratados no presente artigo habitam um país em profunda e duradoura crise econômica, marcado por profundas desigualdades sociais, entre as quais situam-se as de gênero.

\section{Material e Método}

Foi realizado estudo a partir de dados oriundos da Pesquisa Nacional por Amostra de Domicilios (PNAD/IBGE) ${ }^{9}$, de 1986 , sobre morbidade, demanda e utilização de serviços de saúde em dez Estados brasileiros e nas cinco grandes regiōes do país.

A PNAD constitui-se numa das principais fontes de informações demográficas, produzidas em âmbito nacional, com periodicidade regular. Implantada no país em 1967, a partir da década 
de 70, passa a ser realizada anualmente, sempre no último trimestre de cada ano intercensitário".

A pesquisa abrangeu toda a populaçāo residente (moradores presentes e ausentes) em domicilios particulares e coletivos de uma amostra probabilística, obtida em três estágios: no primeiro, foram selecionados municípios (auto-representativos e aqueles sorteados com probabilidade proporcional à população tesidente obtida no Censo Demográfico de 1980; dos municípios da amostra foram sorteados os setores censitárias, também com probabilidade proporcional ao número de domicilios de 1980; desses setores censitários, foram selecionados os domicilios com equiprobabilidade de compor a amostra 9 . Para a expansão da amostra foram utilizados estimadores de razảo cuja variável independente é a projeção da população residente, distribuídos por 11 grupos etários e o tipo de área (regiāo metropolitana e não-metropolitana) ${ }^{9}$.

Através de entrevistas, os moradores presentes responderam a questionário padronizado com perguntas fechadas, por todos os residentes no domicilio. De modo permanente, esse questionário inclui perguntas sobre características demográficas básicas, as relativas à habitação, à mão-de-obra, ao rendimento e à instruçāo. Com periodicidade variável, são incluídos outros temas como a saúde, que constituiu bloco suplementar, em 1981 e em 1986. Nesse último ano, os problemas de saúde foram definidos como a ocorrência de sinais e sintomas de doença nas duas últimas semanas (morbidade referida). As informaçōes sobre demanda e utilização de serviços de saúde também tiveram como período de referência as duas semanas consecutivas anteriores à semana da entrevista?

Foram construídos coeficientes de prevalência de problemas de saúde, de demanda e de utilização de serviços de saúde segundo sexo, sendo todos os dados utilizados provenientes da PNAD $/ 86^{\circ}$ - tanto aqueles relativos aos numeradores, quanto aos denominadores. Os erros amostrais dessas estimativas foram calculados, usando-se o modelo de regressão $\mathrm{y}=\mathrm{AxB}$, onde $x$ é o valor da estimativa, y é o respectivo coeficiente de variação e os parâmetros $A$ e $B$ são fornecidos na publicaçāo consultada. Em decorrência dos dados terem sido trabalhados sob a forma de grandes agregados (Estados e grandes regiōes) os coeficientes de variação dos denominadores situaram-se entre 1,0 e $2,9 \%$ (com média de $1,9 \%$ ) e dos numeradores, entre 2,2 e $8,6 \%$ (com média de 4,9\%), o que resultou $\mathrm{em}$ impacto mínimo nos indicadores. Dessa forma, optou-se pela utilização das estimativas pon- tuais, representadas graficamente, sem maior comprometimento das interpretaçöes dos resultados.

Os coeficientes foram padronizados por idade pelo método direto ${ }^{10}$ e a população padrão foi a brasileira de $1980^{8}$ (ano do último censo com dados já publicados), de modo a permitir a comparação com os resultados de outros estudos compreendendo periodos diferentes.

Como medidas dos diferenciais foram calculadas razões de sexos, ou índices de sobremorbidade, de sobredemanda e de sobreutilização femininas segundo grupos de idade.

\section{Resultados}

As mais altas taxas de morbidade em ambos os sexos foram encontradas em Minas Gerais, no Distrito Federal e em São Paulo (Fig. 1). Também Pernambuco e Bahia apresentaram coeficientes expressivos, especialmente entre as mulheres. A diversidade observada, entretanto, não obedece a um padrão regional nítido.

As maiores taxas de demanda e de utilização de serviços de saúde, em ambos os sexos,

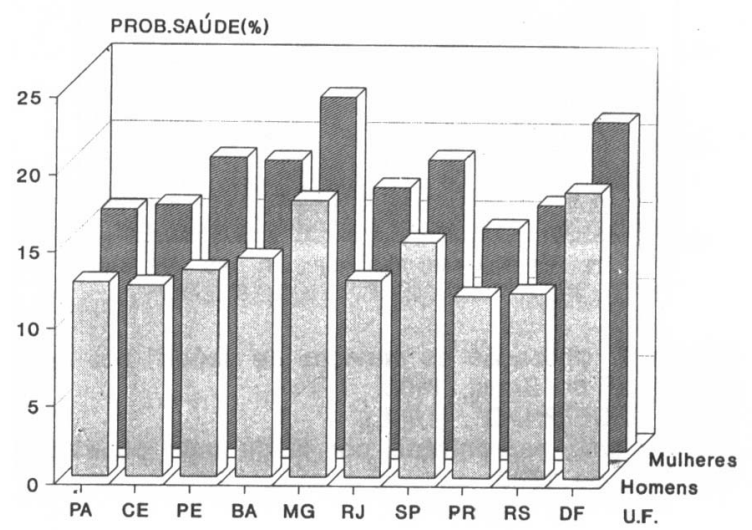

Figura 1. Prevalência de problemas de saúde* por sexo em 10 UF do Brasil, 1986.

Fonte: IBGE/PNAD ${ }^{\circ}$ (1986).

- coeficiente padronizado por idade pela população brasileira de 1980.

foram observadas no Distrito Federal e nos Estados da região sudeste (Fig. 2 e 3).

Em todos os Estados, as mulheres apresentaram maiores prevalências de problemas de saúde que os homens, e os diferenciais por sexo foram praticamente constantes, situando-se em torno de 20 a $30 \%$ de "excesso" feminino de problemas (Fig. 4). As mulheres também referiram maior procura e maior utilização de servi- 


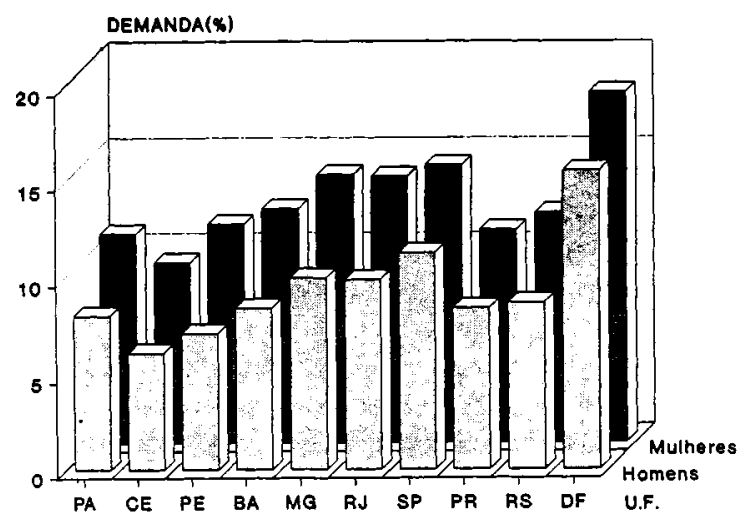

Flgura 2. Demanda por serviçs de saúde* por sexo em 10 UF do Brasil, 1986.

Fonte: IBGE/PNAD'. (1986).

* coeficiente padronizado por idade pela populaçáo brasileira de 1980.

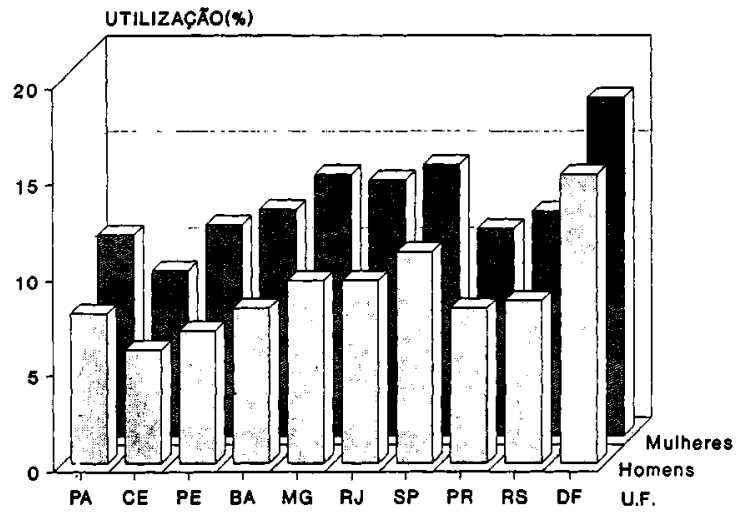

Figura 3. Utilização de serviços de saúde" por sexo em 10 UF do Brasil, 1986.

Fonte: IBGE/PNAD'. (1986).

- coeficiente padronizado por idade pela populacăo brasileira de 1980.

ços que os homens e os maiores diferenciais estiveram nos Estados das regiōes norte e nordeste. Os diferenciais de demanda por sexo foram bem mais acentuados que os de morbidade e os de utilização maiores que os de demanda. Um outro aspecto a ser ressaltado diz respeito à tendência das diferenças regionais acentuaram-se quanto maiores os diferenciais, expressando-se de forma mais marcante na utilização de serviços de saúde.

A análise dos diferenciais de morbidade por idade, que sāo melhor visualizados com os dados agrupados segundo grandes regiōes, revela que estes foram nulos até 14 anos (Fíg. 5). Assumiram os mais altos valores na idade reprodutiva

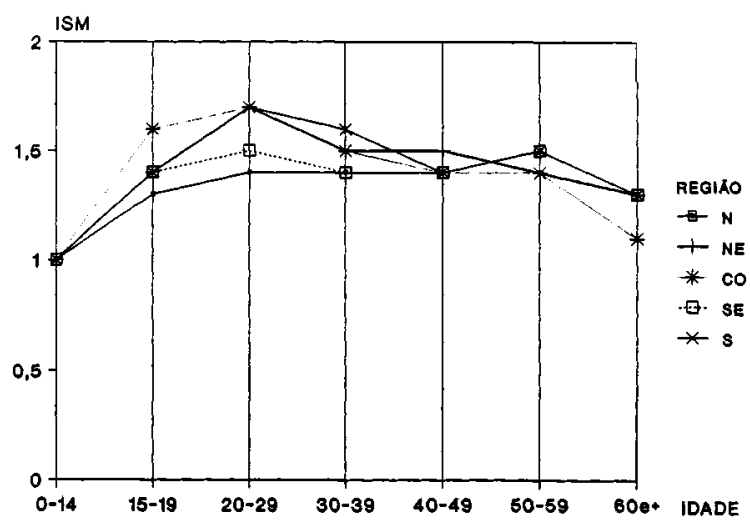

Figura 4. Diferenciais de morbidade, de demanda $\theta$ de utilizaçáo de serviços de saúde" por sexo em grandes regióes do Brasil, 1986.

Fonte: IBGE/PNAD . (1986).

- indices de sobremorbidade, de sobredemanda e de sobreutilização femininas.

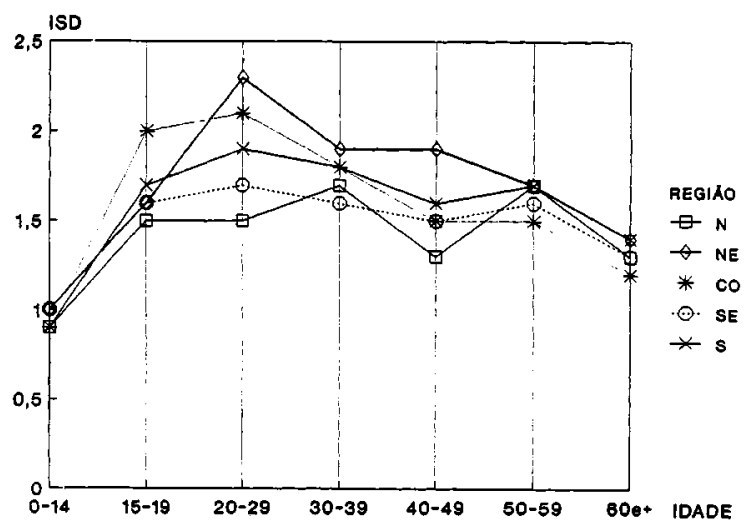

Figura 5. Diferenciais de morbidade por sexo e idade em grandes regióes do Brasil, 1986.

Fonte: IBGE/PNAD . (1986).

das mulheres, com tendência posterior ao decréscimo, exceto no norte e no sudeste. Estas apresentaram curvas bimodais, com o segundo pico entre $\mathbf{5 0}$ a 59 anos, tão alto quanto ou maior que o primeiro. De toda maneira, aos 60 anos e mais, os diferenciais diminuriram, aproximando-se dos valores observados nas demais regiôes.

As curvas dos-diferenciais etários de demanda por serviços de saúde, de um modo geral, assemelharam-se às de morbidade. Apresentaram, contudo, maior variabilidade e valores bem mais altos sobretudo na idade reprodutiva (Fig. 6). Os valores extremos corresponderam a taxas femininas de demanda por serviços de saúde equivalentes a mais que o dobro daquelas observadas em homens da mesma faixa etária. 


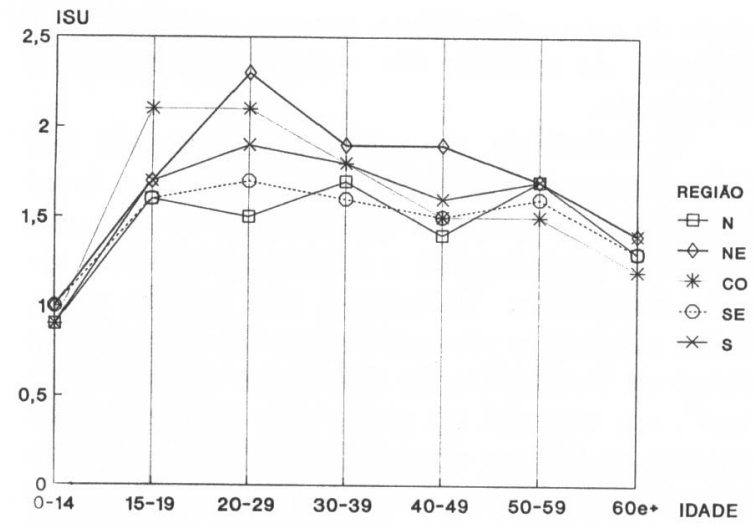

Flgura 6. Diferenciais de demanda por sexo e idade em grandes regiōes do Brasil, 1986.

Fonte: IBGE/PNAD ${ }^{\circ}$ (1986).

Apesar da diversidade entre as regiōes, pode-se identificar a existência de um padrão com as seguintes caracteristicas gerais:

- até os 14 anos, diferenciais nulos, chegando mesmo à inversão nas regiōes centro-oeste, sul e norte, onde se observa uma superdemanda masculina;

- aumento dos diferenciais, a partir de entāo, atingindo-se em todas as regiões, os valores mais altos entre 20 e 29 anos - no nordeste e no centro-oeste estes representaram procura feminina de mais que o dobro da masculina; - tendência ao decréscimo, a partir da faixa de 20 a 29 anos; no sul e no sudeste, as curvas atenuaram-se constituindo quase um platô.

Ressalta-se a curva atípica da região norte, que se configurou bimodal, com um primeiro pico de 30 a 39 anos, mais tardio que as demais, e um segundo pico na faixa de 50 a 59 anos.

Da mesma forma que nas curvas de morbidade, há um decréscimo generalizado a partir dessa faixa etária.

As curvas de diferenciais etários na utilização de serviços de saúde (Fig. 7) praticamente se superpöem às anteriores, com diferenças sutis como, por exemplo, na região centro-oeste, onde o aumento dos diferenciais ocorreu mais precocemente, a partir dos 15 anos, permanecendo os valores inalterados até os 30 anos.

\section{Discussão}

A diversidade observada na prevalência de problemas de saúde, especialmente em mulheres, não pareceu obedecer a um padrão

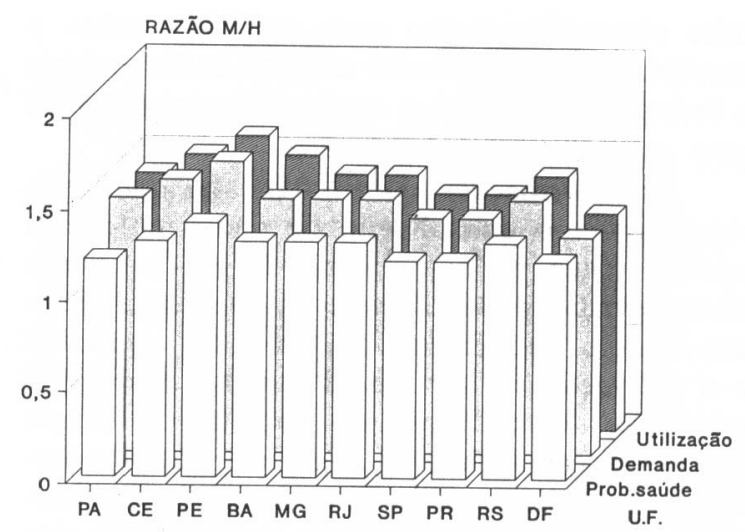

Figura 7. Diferenciais de utilizaça por sexo e idade em 10 UF do Brasil, 1986.

Fonte: IBGE/PNAD." (1986).

regional que pudesse orientar hipóteses explicativas quanto aos seus determinantes. Contudo, $o$ padrão relativo aos indicadores de utilização de serviços provavelmente está relacionado a diferenças quanto à distribuição da oferta que, em última instância determina a procura e o uso de serviços. Os diferenciais por sexo alcançaram seus maiores valores em Estados do norte e nordeste, onde também se situaram as menores taxas de demanda e de utilização, em ambos os sexos. Isso, possivelmente revela, entre outras coisas, uma distribuição desigual da oferta de serviços de saúde, não apenas do ponto de vista quantitativo, mas também qualitativo. Sabidamente, nas regiōes mais carentes de recursos de saúde, a oferta de serviços públicos tende a ser dirigida, de modo prioritário, à população materno-infantil. Ademais, em geral, os serviços funcionam em horários que coincidem com a jornada de trabalho, dificultando mais ainda o acesso da população inserida no mercado formal, em sua maior parte composta de individuos do sexo masculino. As desigualdades regionais estariam assim contribuindo para as diferenças observadas entre os sexos.

A comparação desses resultados com outros estudos apresenta alguns limites em decorrência das especificidades das fontes utilizadas. $\mathrm{Na}$ literatura internacional consultada, as informaçōes são provenientes de inquéritos de diversas naturezas*, realizados ao longo de todo o ano, onde a multiplicidade de variáveis investigadas per-

* Por exemplo, os National Health Interview Survey, National Health Examination Survey, National Hospital Discharge Survey e National Health and Nutrition Examination Survey, nos EUA, c o General Household Survey, na Inglaterra. 
mite obter um quadro mais completo sobre a morbidade, possibilitando análises sofisticadas e a formulação de teorias explicativas consistentes para os fenômenos observados.

As informaçōes da PNAD 9 , ao contrário, representam o consolidado de dados coletados em um único período do ano de 1986, a partir de entrevistas domiciliares com questōes genéricas sobre a ocorrência de problemas, a procura e a utilização de serviços de saúde. Ainda que os meses de realização do trabalho de campo não sejam especialmente atípicos quanto à ocorrência de doenças ou à oferta de serviços, é evidente que a característica de sazonalidade dos fenômenos estudados vai limitar as possibilidades de generalização e de comparabilidade dos resultados.

Entretanto, apesar da ressalva, em todos os Estados estudados, as mulheres apresentaram maior prevalência de problemas de saúde, maior demanda e maior utilização de serviços de saúde, o que é consistente com a literatura internacional.

O maior risco feminino para a maioria das doenças agudas provavelmente está relacionado, em primeiro lugar, às especificidades do trabatho que as mulheres exercem no cuidado da casa e dos filhos. Suas atividades habituais envolvem o contato mais estreito com crianças, que são especialmente sujeitas às infecções; o uso habitual de produtos químicos do lar, ocasionando alergias e lesões dermatológicas; e a adoção de posturas corporais incômodas, levando a problemas músculo-esqueléticos.

A inserção crescente no mercado de trabatho não tem desobrigado as mulheres de suas funções tradicionais, o que implica o acúmulo de tarefas, com maior estresse físico e mental, além dos riscos ocupacionais conseqüentes à dupla inserção. Estudos têm demonstrado que, como decorrência da dupla jornada feminina, o número médio de horas trabalhadas pelas mulheres é superior ao dos homens ${ }^{3}$. O trabalho doméstico, mesmo em sociedades mais desenvolvidas, não tem diminuído substancialmente quanto ao volume, já que houve aumento de sua complexidade e das expectativas relacionadas à limpeza das casas, à lavagem das roupas, ao preparo dos alimentos e à educação dos filhos.

Em nossa sociedade, a sobrecarga de trabatho é particularmente pesada. A ausência de equipamentos sociais, como creches e escolas, conjuga-se à permanência de relações de gênero tradicionais, extremamente assimétricas, sendo atribuída à mulher total responsabilidade no cui- dado da casa e dos filhos, mesmo quando exerce jornadas de trabalho tão extenuantes quanto às do marido.

A existência de maiores diferenciais por sexo durante a idade reprodutiva falaria a favor de diferenças naturais relacionadas à gestação, ao parto, ao puerpério e a outros problemas gineco-obstétricos. Entretanto, em grande parte, essas diferenças são resultantes do intenso processo de medicalização dos ciclos vitais femininos ${ }^{6}$, em especial no nosso país onde são alarmantes os índices de partos cesáreos ${ }^{7}$ e de esterilização de mulheres ainda jovens ${ }^{4}$. Possivelmente, isso se confirma no fato dos diferenciais de demanda e de utilização de serviços segundo sexo serem mais acentuados que aqueles observados quanto à percepção de problemas de saúde, principalmente no período reprodutivo.

$\mathrm{Na}$ interpretação dos resultados, caberia ainda considerar questões de ordem conceitual e metodológica que podem estar contribuindo para as diferenças encontradas.

Uma questão a ser discutida, diz respeito à própria utilizaçăo do conceito problemas de saúde, como expressão de morbidade. Por ser bastante amplo e genérico, esse conceito engloba desde problemas simples, como sintomas inespecíficos ou pequenos acidentes, até afecções graves, como crises hipertensivas, politraumatismos ou meningites, com diferentes graus de confiabilidade quanto ao seu relato ${ }^{11,2}$. Além disso, o relato de problemas percebidos pelos entrevistados não expressa doenças clinicamente definidas, mas a construção de experiências de adoecimento, que se dá de modo diferente entre os sexos, entre classes e entre culturas ${ }^{6}$. Isso poderia explicar a existência de diferentes prevalências entre Estados de uma mesma região, com características comuns quanto às condições de vida de suas populações e mesmo com padrões de mortalidade semelhantes, mas que apresentam aspectos culturais diversos.

Uma questão metodológica da maior relevância diz respeito ao fato das informações na PNAD serem obtidas também através de proxirespondentes, na maioria das vezes mulheres que se encontram em casa no momento da visita . Por razões óbvias, a freqüente preponderân-

\footnotetext{
* Esté procedimento tem sido bastante utilizado, como forma de diminuir os custos das pesquisas, já que um ou mais individuos presentes respondem por todos aqueles que, embora elegiveis para compor a amostra, estão ausentes na entrevista. Ás mulheres têm sido atribuido maior conhecimento sobre os problemas de saúde da famillia pelo papel central que ocupam no cuidado dos fillhos e dos matidos. Além disso, são mais facilmente localizáveis e mais colaborativas, consequientemente contribuindo para o barateamento da coleta de dados.
} 
cia de um dos sexos, entre aqueles adotados como proxi-respondentes, faz com que esta seja uma estratégia limitada para o estụdo de diferenças de gênero, principalmente porque a estratificação dos dados segundo a característica de ter sido ou não pessoalmente entrevistado não foi viável na análise dos resultados por absoluta impossibilidade de se resgatar essa informação.

De um modo geral, existe tendência a haver maior fidedignidade quando as informaçōes dizem respeito a experiências pessoais do que as referentes àquelas ocorridas com terceiros ausentes. Quanto mais subjetiva a questão investigada, menos precisa será a informação ${ }^{11,15}$. E possivel, portanto, que os relatos sobre a procura e a utilização de serviços apresentem melhor qualidade do que os que se referem à morbidade percebida, embora não se possa avaliar em que medida as mulheres tomam conhecimento dos contatos estabelecidos pelos diversos familiares com os serviços de saúde, excetuando-se evidentemente seus próprios filhos menores.

$O$ alto grau de generalidade do conceito de morbidade adotado possibilita ainda a influência de diferenças de gênero na valorização dos eventos. Não apenas o relato dos problemas é diferenciado - com as mulheres tendendo a falar mais sobre seus sintomas e a admitir mais facilmente que estão doentes -, como a percepção dos problemas do parceiro tenderia a ser diferente. Além disso, a forma de reagir a tensões do cotidiano é diversa: as mulheres costumam expressar ansiedade e tensão através de sintomas físicos, enquanto os homens manifestam comportamentos que, habitualmente, não são considerados como doenças - fumando, bebendo, reagindo com violência, entre outros ${ }^{6,17}$. Estes comportamentos, todavia, são conhecidos fatores de risco para acidentes e para doenças crônicas, como o infarto agudo do coração e o câncer de pulmão, responsáveis por grande parte dos óbitos masculinos.

Esta primeira aproximação do problema em questão apenas aponta a necessidade de novos estudos que permitam indicações mais precisas, tanto sobre o quadro atual e seus determinantes, quanto sobre possiveis perspectivas futuras.

No Brasil, a PNAD constitui-se na única fonte de informaçōes sobre saúde de base populacional, com abrangência nacional. Anteriormente ao ano de 1986, apenas uma outra vez, em 1981, sua realização incluiu um bloco suplementar sobre saúde. $O$ inquérito anterior, embora mais abrangente, teve problemas metodológicos sérios, inclusive na própria formulação dos quesitos sobre saúde ${ }^{*}$, ocasionando um alto grau de subestimação das taxas de prevalência de problemas e de utilização, o que pode ser verificado em estudo regional ${ }^{3}$. Isso, evidentemente, inviabiliza a identificação de tendências temporais na realidade brasileira, o que seria extremamente relevante diante das profundas transformações que vêm ocorrendo na situação social da mulher. Nos EUA, por exemplo, mudanças relativas à diversidade de gênero no acesso à tecnologia diagnóstica e terapêutica, bem como no próprio lugar social das mulheres, podem estar contribuindo para a ocortência de modificações nas tradicionais diferenças de sexo dos indicadores de saúde. E, embora permaneça existindo um "excesso" feminino de relato de problemas de saúde e de uso dos serviços, os diferenciais têm diminuído quanto às incapacidades decorrentes de doenças agudas e crônicas, e mesmo quanto à ocorrência deste último grupo de problemas $^{17}$.

No presente estudo, observou-se que, relativamente, os diferenciais são menores nos Estados mais desenvolvidos do país. Se essas diferenças regionais podem expressar algo sobre tendências temporais, é possivel supor que, mantidas as condiçōes atuais de aumento da inserção da mulher no mercado de trabalho e conseqüentes modificações de seu papel tradicional, as diferenças entre os sexos podem diminuir, seja na percepção dos problemas, seja na utilizaçāo de serviços. Há que considerar, todavia, que essa inserção se dá de modo bastante diferenciado dos homens e que as mudanças que vêm ocorrendo não significam necessariamente um apagamento da assimetria existente nas relações de gênero.

Finalmente, o impressionante fenômeno de medicalização dos ciclos reprodutivos femininos, que se expressa tanto nos altos índices de partos cesáreos, quanto na esterilização de amplos contingentes de mulheres jovens, têm efeitos ainda imponderáveis sobre a saúde e sobre a próptia construção do processo de adoecimento pelas mulheres.

Decerto, a investigação sobre essas questões é da maior relevância, particularmente diante da necessidade de reorganização do sistema de saúde de modo eqüânime e universal.

\footnotetext{
* Como principal limite, pode ser apontado o fato de se tomar como periodo de referência o intervalo de duas semanas compreendido entre datas pré-fixadas, ao invés daquelas imediatamente antecedentes ao momento da entrevista; assim, foram colhidas informações relativas a diferentes momentos passados, o que evidentemente tem repercussōes quanto à possibilidade de bias de memória, em especial, quanto menos graves e menos objetivos forem os eventos.
} 


\section{Agradecimentos}

A Letícia C.C. Nobre, mestranda em Saúde Comunitária da UFBA, pela sua participaçāo na coleta e no processamento dos dados deste estudo; ao Eduardo Motta, professor do Departamento de Medicina Preventiva da UFBA, pelo apoio no tratamento estatístico dos dados.

AQUINO, E.M.L. et al. [Gender and health in Brazil: considerations based on the National Household Sampling Survey]. Rev. Saúde públ., S. Paulo, 26: 195-202, 1992. As in the principal industrial countries, Brazilian women have lived longer than men. However, paradoxically, women present higher morbidity indicators than men. Knowledge of the Brazilian pattern regarding this matter could be a useful contribution to an understanding of their determinants in our specific reality, as well as enabling us to foresee future trends that would make it possible to plan adjustment in the health system. A morbidity study based on data from the National Household Sample Survey (PNAD/IBGE), was undertaken in ten Brazilian states in 1986 with this in view. Coefficients of the prevalence of perceived morbidity, demand for and utilization of health services according to sex, standardized by age and using the direct method, were built up. As a measurement of the differentials, sex ratios were calculated. The excess of perceived morbidity in women was constant in all the regions. The sex differential in the utilization of health services showed regional variations, suggesting a relationship with the health services supply. Sex differentials were not observed in childhood; the highest values were found during the woman's reproductive period, decreasing sharply after 60 years of age. The pattern is very similar in all regions. In the present study, the findings could be partially explained by the methodology adopted, but they are similar to the findings reported in other countries. The intense transformations in the reproductive pattern and in the social status of Brazilian women probably have a considerable impact on the health status and on the recourse to health services, not as yet evaluated. The development of more specific studies that may contribute to the reorganization of a fair and universal health system is recommended.

Keywords: Women's health. Morbidity. Health services needs and demand, trends.

\section{Referências Bibliográficas}

1. ANDERSEN, R \& ANDERSEN, O.W. A decade of health services. Chicago, University of Chicago Press, 1967. Apud Nathanson, C.A. Sex, illness and medical care: a review of data, theory and method. Soc. Sci. Med., 11: 13-25, 1977.
2. AQUINO, E.M.L; MENEZES, G.M.; AMOEDO M.B.E.; NOBRE, L.C.C. Mortalidade feminina no Brasil: sexo frágil ou sexo forte? Cad Saúde públ., Rio de Janeiro, 7(2): 174-89, 1991.

3. BARROSO, C. Trabalho e saúde da mulher. Rev. bras. Saúde ocup., 38(10): 7-11, 1982.

4. BERQUÓ, EA. A esterilização feminina no Brasil hoje. In: Ministério da Justiça. Conselho Nacional dos Direito da Mulher (CNDM). Quando a paciente ${ }^{2}$ mulher. Brasilia, 1989. p. 79-84.

5. CARVALHO, F.M.; SILVANY NETO, A.M.; PAIM, J.S.; MELO, A.M.C. de; AZARO, M. da G.A. Morbidade referida e utilização de consulta médica cm cinco populações do Estado da Bahia. Ciênc. Cult., 40: 853-8, 1988.

6. CLARKE, J. Sexism, feminism and medicalism: a decade review of literature on gender and illness. Soc. Hlth Illn., 5(1): 62-82, 1983.

7. FAÚNDES, A. \& CECATTI, J.G. A operação cesárea no Brasil: incidência, tendências, causas, consequuências e propostas de ação. Cad Saúde públ., Rio de Janeiro, 7(2): 150-73, 1991.

8. FUNDAÇẢo IBGE. Censo demográfico: dados gerais, migração, instrução, fecundidade, mortalidade: Brasil, 1980. Rio de Janeiro, 1982. v. 1, t. 4, $\mathrm{n}^{2} 1$. (Recenseamento Geral do Brasil, 1980).

9. FUNDAÇẢo IBGE. Pesquisa Nacional por Amostra de Domicilios: Brasil, 1986. Rio de Janeiro, 1989. Suplemento $\mathrm{n}^{2}$ 3: Acesso a serviços de saúde.

10. KIRWOOD, B.R. Essentials of medical statistics. London, Blackwell Scientific Publ., 1988. p. 112-4.

11. KROEGER, A. Health interview surveys in developing countries: a review of methods and results. Int. J. Epidem., 12: 465-81, 1983.

12. MECHANIC, D. \& NEWTON, M. Some problems in the analysis of morbidity data. J. chron. Dis., 18: 569-80, 1965.

13. A MULHER brasileira: estatística de saúde. Radis/Dadas FIOCRUZ, Rio de Janeiro, 4: 1-24, 1986.

14. NATHANSON, C.A. Illness and femine role: a theoretical review. Soc. Sci. Med, 9: 57-62, 1975.

15. NATHANSON, C.A. Sex, illness and medical care: a review of data, theory and method. Soc. Sci. Med. 11: 13-25, 1977.

16. SCOTT, J. Gênero: uma categoria útil para análise histórica Educ. Real., Porto Alegre, 16(2): 5-22, 1990.

17. VERBRUGGE, L.M. Females and illness: recent trends in sex differences in the Unites States. $J$. Hlth soc. Behav., 17: 387-403, 1976.

18. VERBRUGGE, L.M. Sex differentials in health. Publ. Hlth Rep., 97: 417-37, 1982.

19. WINGARD, D.L The sex differential in mortality rates: demographic and behavioral factors. Amer. $J$. Epidem., 115: 205-16, 1982.

20. WINGARD, D.L. The sex differential in morbidity, mortality and lifestyle. Ann. Rev. publ. Hlth, 5: 433-58, 1984.

21. WINGARD, D.L; SUAREZ, L.; BARRETT-CONNOR, E. The sex differential in mortality from all causes and ischemic heart disease. Amer. J. Epidem., 117: 165-72, 1983. 